PREPARED FOR THE U.S. DEPARTMENT OF ENERGY, UNDER CONTRACT DE-AC02-76CH03073

PPPL-3998

PPPL-3998

UC-70

Beam Anisotropy Effect on Alfvén Eigenmode

Stability in ITER-like Plasma

by

N.N. Gorelenkov, H.L. Berk, and R.V. Budny

August 2004

$\left.\stackrel{M}{M}\right|_{\substack{\text { PRInCETON PLASIMA } \\ \text { PHYSICS LABORATORY }}} ^{D}$

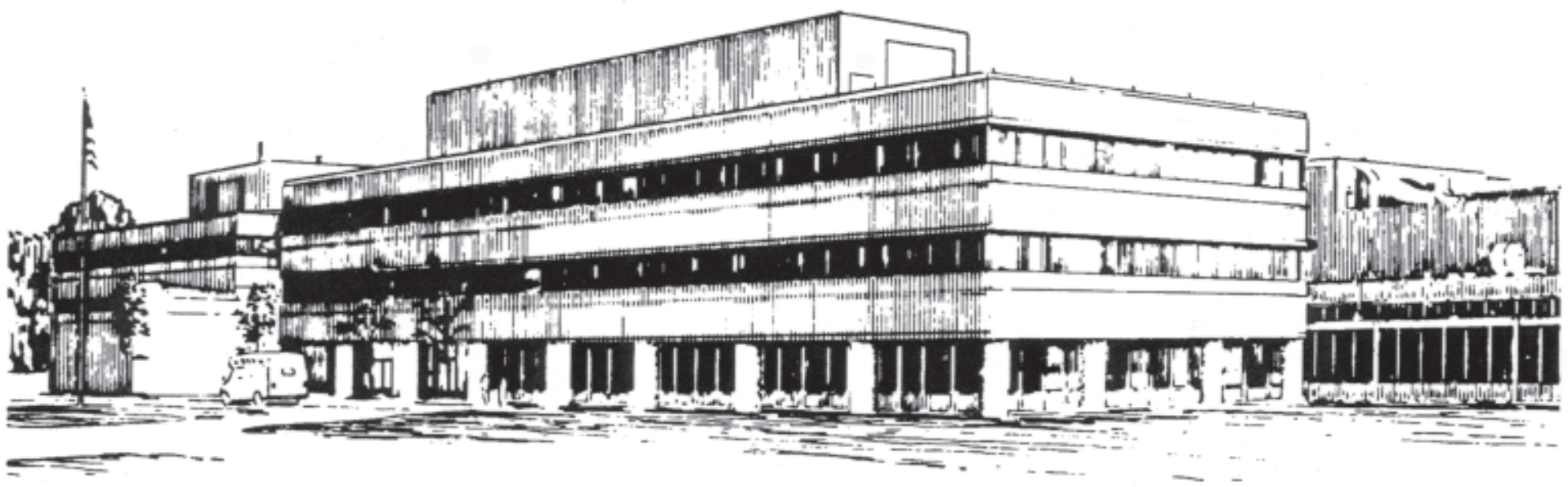

PRINCETON PLASMA PHYSICS LABORATORY PRINCETON UNIVERSITY, PRINCETON, NEW JERSEY 


\section{PPPL Reports Disclaimer}

This report was prepared as an account of work sponsored by an agency of the United States Government. Neither the United States Government nor any agency thereof, nor any of their employees, makes any warranty, express or implied, or assumes any legal liability or responsibility for the accuracy, completeness, or usefulness of any information, apparatus, product, or process disclosed, or represents that its use would not infringe privately owned rights. Reference herein to any specific commercial product, process, or service by trade name, trademark, manufacturer, or otherwise, does not necessarily constitute or imply its endorsement, recommendation, or favoring by the United States Government or any agency thereof. The views and opinions of authors expressed herein do not necessarily state or reflect those of the United States Government or any agency thereof.

\section{Availability}

This report is posted on the U.S. Department of Energy's Princeton Plasma Physics Laboratory Publications and Reports web site in Fiscal Year 2004. The home page for PPPL Reports and Publications is: http://www.pppl.gov/pub_report/

DOE and DOE Contractors can obtain copies of this report from:

U.S. Department of Energy

Office of Scientific and Technical Information

DOE Technical Information Services (DTIS)

P.O. Box 62

Oak Ridge, TN 37831

Telephone: (865) 576-8401

Fax: (865) 576-5728

Email: reports@adonis.osti.gov

This report is available to the general public from:

National Technical Information Service

U.S. Department of Commerce

5285 Port Royal Road

Springfield, VA 22161

Telephone: $1-800-553-6847$ or

(703) $605-6000$

Fax: (703) 321-8547

Internet: http://www.ntis.gov/ordering.htm 


\title{
Beam anisotropy effect on Alfvén Eigenmode stability in ITER-like plasma
}

\author{
N. N. Gorelenkov ${ }^{\dagger}$, H. L. Berk ${ }^{\ddagger}$, R. V. Budny ${ }^{\dagger}$ \\ $\dagger$ Princeton Plasma Physics Laboratory \\ P.O. Box 451, Princeton, NJ 08543-0451. \\ ‡IFS, Austin, Texas.
}

\begin{abstract}
This work studies the stability of the toroidicity-induced Alfvén Eigenmodes (TAE) in the proposed ITER burning plasma experiment, which can be driven unstable by two groups of energetic particles, the $3.5 \mathrm{MeV}$ $\alpha$-particle fusion products and the tangentially injected $1 \mathrm{MeV}$ beam ions. Both species are super-Alfvénic but they have different pitch angle distributions and the drive for the same pressure gradients is typically stronger from co-injected beam ions as compared with the isotropically distributed $\alpha$-particles. This study includes the effect of anisotropy of the beam ion distribution function on TAE growth rate directly via the additional velocity space drive and indirectly in terms of the enhanced effect of the resonant particle phase space density. For near parallel injection TAEs are marginally unstable if the injection aims at the plasma center where the ion Landau damping is strong, whereas with the off-axis NBI the instability is stronger with the growth rate near $0.5 \%$ of TAE mode frequency. In contrast, for perpendicular beam injection TAEs are predicted to be stabilized in nominal ITER discharges.

In addition, the effect of TAEs on the fast ion beta profiles is evaluated on the bases of a quasi-linear diffusion model which makes use of analytic expressions for the local growth and damping rates. These results illustrate the parameter window that is available for plasma burn when TAE modes are excited.
\end{abstract}




\section{INTRODUCTION}

The Toroidal Alfvèn Eigenmodes (TAE's) [1-4] destabilized by fast ions can cause significant difficulties for the containment of energetic alpha particles in fusion energy generating tokamak experiments. Experimentally it is also known that various conditions can be obtained with varying degrees of severity. The modes can be stable, or unstable where energetic particle loss is insignificant $[5,6]$. However it was established that in the presence of a strong enough energetic particle energy density these modes will induce large losses of fast particles.

In the proposed ITER fusion experiment, $1 \mathrm{MeV}$ neutral beam injection (NBI) is planned for current drive and auxiliary heating [7]. The neutral beams will be injected in the direction of the plasma current and will create an anisotropic distribution of super-alfvénic beam ions, which will drive TAEs unstable with partial growth rate contributions that are comparable to the contribution from fusion alphas [8]. In this reference it was found that for a nominal ITER experiment operating, with the central temperature at $20 \mathrm{keV}$, the plasma would be marginally unstable to alpha particle TAE instability. An initial study of the beam ion contribution to the TAE drive showed that the beams were an additive contribution to the alpha particle instability. In addition it was shown that the anisotropy in the beam ion distribution function results in an additional velocity space drive. Since the beam ion contribution is significant it is important to carefully evaluate this mechanism and, if possible, find a TAE stable plasma parameter domain.

The purpose of the first part of the paper is to determine the effect of beam ions with an anisotropic distribution function on the linear instability of TAE's under burning plasma conditions. We use the perturbative NOVA/NOVA-K codes $[9,10]$, which were successfully employed to predict the thresholds and frequencies of medium- $n$ TAE instabilities in various exper-

iments including the beam heated deuterium-tritium experiments on TFTR $[11,12]$. Recently NOVA/NOVA-K codes were successfully used to explain high- $n$ odd core localized TAEs observed in ICRH JET discharges [13]. In these codes the procedure used is to first obtain the lowest order mode structure using the ideal MHD NOVA code which neglects damping and drive sources. Then the NOVA-K code incorporates these sources through a perturbative procedure by evaluating corresponding integrals over the known eigenfunctions. At moderate- to high- $n$ 's the lowest order TAE mode often extends over the entire minor radius. When the continuum is not excited in the regions where amplitude of the eigenmode is significant, the global structure of the TAE mode can then be used as a lowest order approximation to the eigenmode. Then the beam ion drive 
and other numerous damping mechanisms are incorporated in a perturbative manner to predict stability. All equilibrium plasma parameters and profiles are obtained by employing the transport code TRANSP [14]. In this paper we analyse details of TAE stability for one of the most unstable toroidal mode numbers, $n=10$, to examine the sensitivity to NBI injection characteristics, such as geometry and energy.

One can expect TAE unstable plasmas to still produce tolerable reactor performance if only moderate alpha transport is induced that primarily redistributes the alpha particle profile inside the plasma rather than cause direct losses to the plasma facing walls. The strength of the redistribution should depend upon how far above the marginal threshold the modes are excited, among other factors. We propose a model in Section VI in which the local TAE stability theory is applied to reconstruct the selfconsistent fusion alphas beta profiles on the bases of a quasi-linear diffusion model. This model makes use of expressions for the local growth and damping rates and calculates the relaxed alpha particle profiles by imposing the particle conservation condition for the steady state.

\section{ITER PLASMA MODELING}

Equilibrium plasma profiles of the ITER nominal discharge are calculated by the transport code TRANSP [14] and are presented in Fig. 1, where the plasma density, safety factor, alpha particle and beam ion beta profiles are plotted as functions of minor radius variable $r / a \equiv \sqrt{\Phi}$, where $\Phi$ is the toroidal magnetic field flux, normalized to 1 at the plasma edge and 0 at the plasma center. Other plasma parameters we use are: a major plasma radius of the geometrical center of $R_{0}=$ $6.2 m$, a minor radius of the last magnetic surface of $a=2 m$, a deuterium negative NBI injection power $P_{N B I}=33 M W$ at an energy $E_{b 0}=1 M e V$, a vacuum magnetic field at the geometrical axis of $B_{0}=5 T$, a total central beta value of $\beta_{0}=6.7 \%$ and ion and electron temperatures in the center at $T_{i 0}=19.5 \mathrm{keV}$ and $T_{e 0}=23.5 \mathrm{keV}$ respectively. The injection geometry is taken from the ITER design [15] and is slightly different from the one used in previous calculations [8, 14], which results in up to approximately $50 \%$ lower fast ion beta values. These parameters were input

to TRANSP simulations where NBI was applied tangentially $0.55 \mathrm{~m}$ below the magnetic axis. Note that the resulting safety factor profile has a region of lower shear at $r / a \sim 0.5$ because the proposed NBI and ICRH modify the current density profile. The low shear region turns out to be an important for the stability considerations of global TAEs. 
This set of plasma parameters corresponds to one of the more unstable cases we have considered. In this case the beam injection produces hollow beam beta profile and a strong pressure gradient in the region $r / a \sim 0.5$, which is the region where the damping is relatively weak, which allows TAEs to be excited (more details are given below).
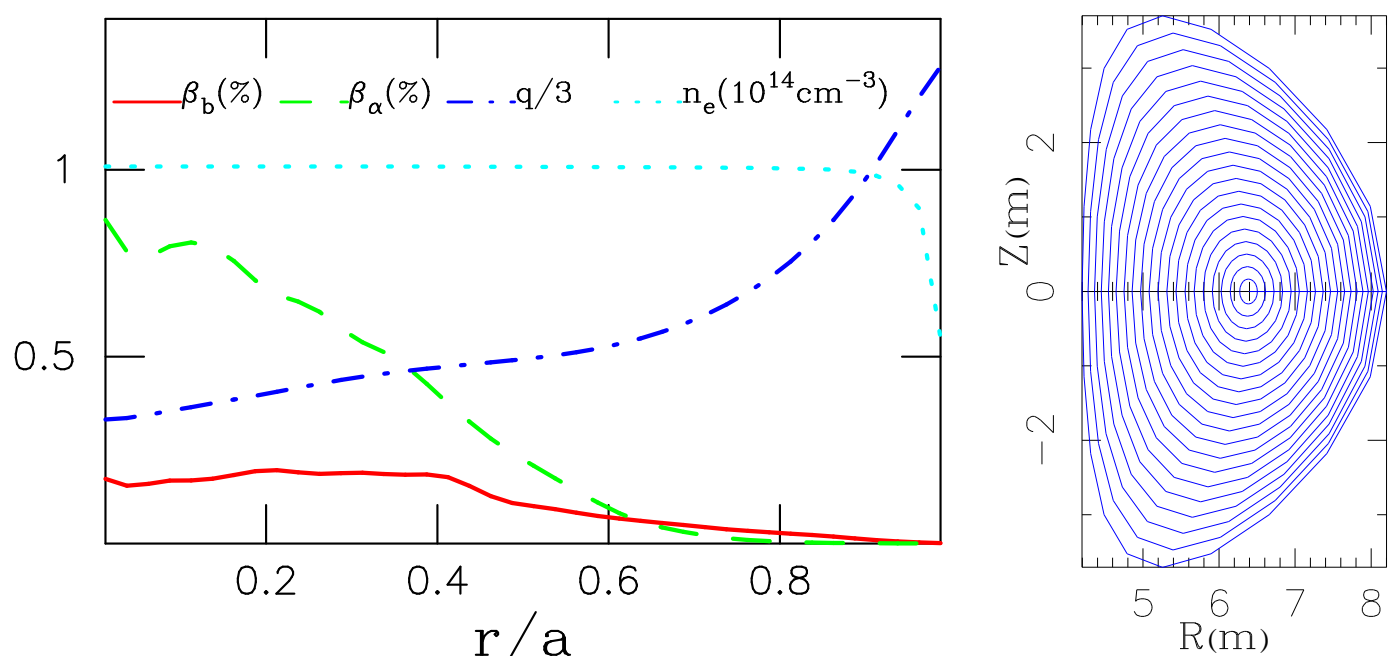

Figure 1: Plasma profiles for the ITER plasma equilibrium (left plot) and corresponding magnetic surfaces (right plot) as predicted by TRANSP code. On the left plot shown are the beam beta $\beta_{b}(\%)$, the fusion alphas beta $\beta_{\alpha}(\%)$, safety factor $q$ and the electron density $n_{e}\left(10^{14} \mathrm{~cm}^{-3}\right)$.

In addition to case (1) with $0.55 \mathrm{~m}$ off-axis NBI, three other injection configurations have been studied (cases (2), (3), and (4)) as shown in figure 2. In all these cases the plasma beta was fixed at a beta value that is characteristic of the value expected by the constraint that the discharge be MHD stable. Cases (2) and (3) are for fixed $T_{i 0}=19.5 \mathrm{keV}$, with case (2) with on-axis injection while case (3) is for $0.35 \mathrm{~m}$ off-axis. The third additional case is for a higher ion temperature, $T_{i 0}=25.3 \mathrm{keV}$ and on-axis NBI.

\section{A. NBI ion anisotropic distribution function}

Since the beam injection creates an anisotropic beam ion distribution function, one needs to model it carefully due to its potentially strong effect on TAE stability. In this study we develop a model for the co-injected (as proposed for the ITER design) beam ion slowing down distribution function and a Gaussian in pitch angle dependence centered at some average value, $\chi_{0}$, where 


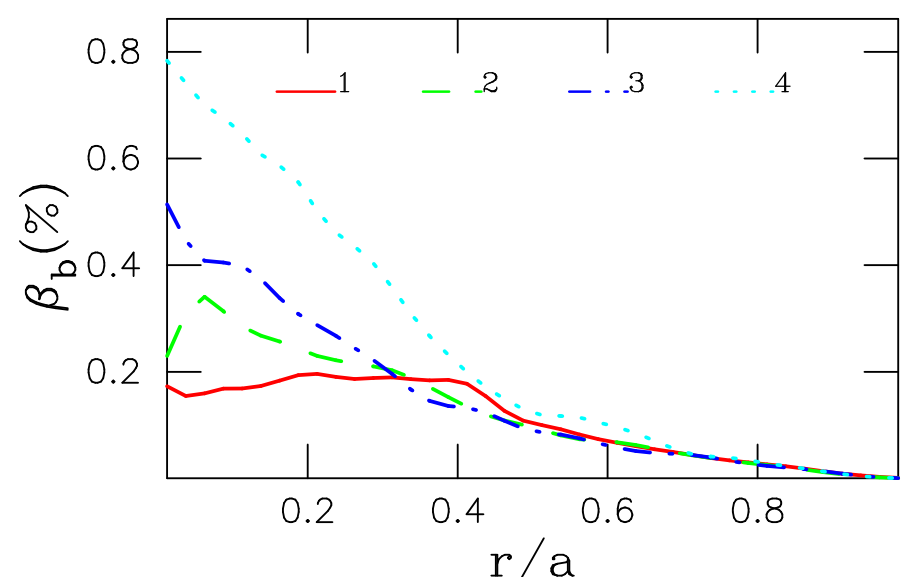

Figure 2: Equilibrium beam beta profiles in four ITER plasmas under the consideration. Curves 1,2, and 3 are for the plasma ion temperature $T_{i 0}=19.5 \mathrm{keV}$, and injection geometry aiming with vertical shift $0.55 \mathrm{~m}$, $0.35 \mathrm{~m}$, and $0 \mathrm{~m}$, respectively, with the respect to the equatorial plane. Curve 4 corresponds to the on-axis NBI at plasma temperature $T_{i 0}=25.3 \mathrm{keV}$.

$\chi \equiv v_{\|} / v$ is the pitch angle taken at the equatorial plane at the low field side of the plasma cross section. We will see that this model captures the main physics relevant for AE stability analysis and which appears to be consistent with the results obtained from the Monte-Carlo post-processor of the TRANSP code [14].

If NBI generates a narrow pitch angle distribution it can be modeled in the following form:

$$
f_{b}=\frac{f_{\chi}(\chi, v, r)}{v^{3}+v_{*}^{3}} \frac{C(r)}{\delta \chi(v . r)}, \text { if } v<v_{b 0},
$$

and $f_{b}=0$ if $v>v_{b 0}$, where $f_{\chi}(\chi, v, r) \equiv e^{-\left(\chi-\chi_{0}\right)^{2} / \delta \chi(v, r)^{2}}, \delta \chi^{2} \ll \chi_{0}^{2}$ is assumed. In this formula $\delta \chi$ is the width of the pitch angle distribution function, $v_{*}$ is the critical velocity at which the ion collisional drag becomes comparable with the drag on electrons, $v_{b 0}$ is the injection velocity, $C(r)$ is the normalization function. As we take the ions to be injected in the passing region parallel to the current flow $\chi_{0}>0$, the trapped and the and passing particles that flow in the direction opposite to the plasma current only appear in the plasma due to Coulomb collisional scattering. Special care is then needed in how we account for such particles in the distributions we study. To high accuracy this distribution function obeys the steady solution in terms of the integrals of motion (IOM) of the drift kinetic equation, e.g. see [16]). The selection of the equation needs special consideration to be compatible with the collisional slowing down process. In general this problem needs to be solved numerically, which is a difficult problem beyond the scope of this paper. Instead we select 
a distribution functional form that qualitatively captures the effect of collisions. Note that $f_{b}$ is a function of a particle's IOM, since $\chi$ at the low field side can be expressed in terms of IOM. The pitch angle width $\delta \chi(v, r)$ changes as fast ions slow down due to the Coulomb scattering [17, 18]. The form of $\delta \chi(v, r)$ can be straightforwardly derived by noting that while beam ions slow down they experience Coulomb scattering with a pitch angle scattering diffusion coefficient given by $D_{\chi \chi} \simeq v_{*}^{3} / v^{3} \tau_{s e}$. The problem can be reduced to the following equation

$$
\frac{\partial f}{\partial \tau}=D_{\chi \chi} \frac{\partial^{2} f}{\partial \chi^{2}}+\delta\left(\chi-\chi_{0}\right) \theta\left(\tau-\tau_{0}\right)
$$

where $d \tau=d v v^{2} /\left(v^{3}+v_{*}^{3}\right), \theta$ is the step function, and $\tau_{0}=\tau_{\mid v=v_{0}}$. Here we assumed that the injection is at a single pitch angle $\chi_{0}$. Simultaneously the particle velocity is determined from the velocity drag and it is found to satisfy

$$
v^{3}=\left(v_{*}^{3}+v_{b 0}^{3}\right) e^{-3 t / \tau_{s e}}-v_{*}^{3},
$$

where $\tau_{s e}$ is the ion slowing down time due to collisions with electrons. At a given time the pitch angle width is broadened according to equation $\delta \chi^{2}=\delta \chi_{0}^{2}+\int_{0}^{t} D_{x x} d t$, where $\delta \chi_{0}$ is the initial width due to ion finite orbit width (FOW) and plasma aspect ratio effects. Thus it follows that the width is given by

$$
\delta \chi^{2}(v, r)=\delta \chi_{0}^{2}-\frac{1}{3} \ln \left[\frac{v^{3}\left(1+v_{*}^{3} / v_{b 0}^{3}\right)}{v^{3}+v_{*}^{3}}\right] .
$$

The center of the pitch angle Gaussian distribution function, $\chi_{0}$, is computed by taking the first moment of the numerical distribution function generated by TRANSP. In the case analyzed we obtained $\chi_{0} \simeq 0.8$ near the region of interest. i.e. $r / a \simeq 0.5$ and $\chi_{0}$ does not change significantly in the vicinity of the TAE location.

Figure 3(a) shows contours of constant $f$ for the distribution function generated by TRANSP and the numerical pitch angle distribution widths $\delta \chi^{2}$, are shown in figure 3(b) along with the widths fitted to the analytic results,

$$
\delta \chi^{2}(v, r)=\delta \chi^{2}(v)=0.015-\frac{1}{3} \ln \left[\frac{\left(v / v_{b 0}\right)^{3} 1.275}{\left(v / v_{b 0}\right)^{3}+0.275}\right]
$$

In making a fit to the numerical width we used velocity parametric dependence whereas other coefficients were computed. Here the minor radius dependence can be neglected within the mode location. Eq.(5) implies that $v_{*}^{2} / v_{b 0}^{2}=0.423$, which is typical predicted value for ITER. 

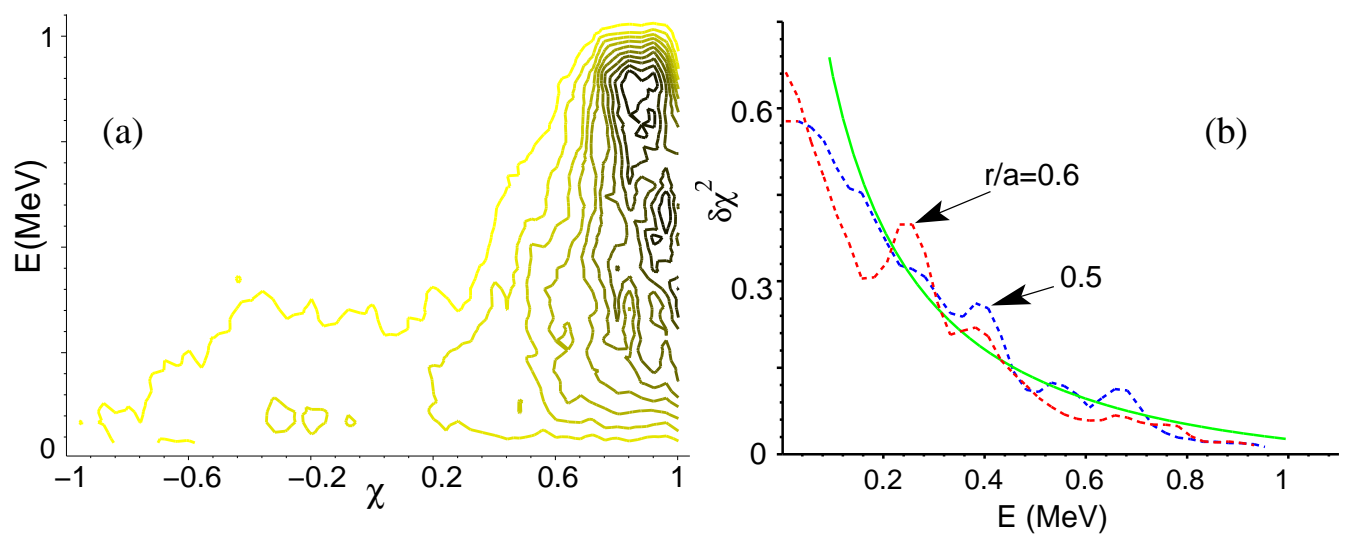

Figure 3: Contours of constant distribution function of beam ions (a) taken at the low field side of the plasma cross section and averaged over $0.5<r / a<0.6$. Figure (b) shows the pitch angle width of the beam ion distribution function versus particle energy at two minor radii $r / a=0.5$ and 0.6 . These plots are compared favorably with the widths resulting from the analytic model (dashed curve) given by Eq.(1).

If the pitch angle width becomes large, the above $\chi$ dependence of the distribution function needs to be modified. Consider three regions: (I) positive passing ions $\chi_{s+}<\chi<1$, where $\chi_{s+}$ is the pitch angle at the separatrix between the co-moving (with respect to the plasma current) passing and the trapped ones; (II) trapped ions $\chi_{s_{-}}<\chi<\chi_{s_{+}}$; and (III) negative passing ions $-1<\chi<\chi_{s-}$, where $\chi_{s-}$ refers to the separatrix between the counter-moving passing and trapped ions. In the case of a large aspect ratio plasma $\chi_{s+}=-\chi_{s_{-}}=\sqrt{2 \varepsilon}$. If we relax the boundary condition at $\chi=-1$ we can then satisfy the boundary condition for the particle flux near the separatrix by the method of images. The flux condition requires that the fluxes in and out the separatrix region are equal:

$$
\frac{\partial f\left(\chi_{s+}+\varepsilon\right)}{\partial \chi} \tau_{+}=\frac{\partial f\left(\chi_{s-}-\varepsilon\right)}{\partial \chi} \tau_{-}+\frac{\partial f\left(\chi_{s+}-\varepsilon\right)}{\partial \chi} \tau_{+}+\frac{\partial f\left(\chi_{s-}+\varepsilon\right)}{\partial \chi} \tau_{-},
$$

where $\tau_{+}$is the part of trapped particle drift orbit period containing particle positive parallel velocity, and $\tau_{-}$corresponds to the rest of particle orbit. In the above condition for fluxes we also assumed that the separatrix dependence on velocity is weak. In the following we present the derivation for the case of large aspect ratio plasma without fast ion orbit effects, in which $\tau_{-}=\tau_{+}$, the flux condition equation reads $f^{\prime}\left(\chi_{s+}+\varepsilon\right)=f^{\prime}\left(\chi_{s-}-\varepsilon\right)+2 f^{\prime}\left(\chi_{s+}-\varepsilon\right)$, and we require that the trapped ion distribution function inside the region II is even in $\chi$.

Since the possible pitch angle range is bounded by $|\chi|<1$, image particle sources can be introduced in order not to have diffusive fluxes at the boundaries. In that case the distribution 
function satisfies the physical requirement of zero derivative at the boundary

$$
f_{\chi p}(\chi)=\phi(\chi) \equiv e^{-\left(\chi-\chi_{0}\right)^{2} / \delta \chi^{2}}+e^{-\left(\chi-2+\chi_{0}\right)^{2} / \delta \chi^{2}}
$$

where two terms correspond in the right hand side correspond to curves 1 and 2 shown in Fig. 4. Since particles are diffusing out of the region I an image sink needs to be introduced. In regions II and III image sources at half the actual source strength adequately represent the solution. Thus we finally obtain

$$
f_{\chi}=\left\{\begin{array}{cc}
\phi(\chi)-\frac{1}{2} \phi\left(2 \chi_{s+}-\chi\right), & \text { region } I \\
\frac{1}{2} \phi(-\chi)+\frac{1}{2} \phi(\chi), & \text { II } \\
\frac{1}{2} \phi\left(2 \chi_{s+}+\chi\right), & \text { III }
\end{array} .\right.
$$

The procedure described is valid for a broad range of pitch angles width and the truncation only becomes invalid at late times when it is necessary to account for collisional fluxes resulting from multiple reflections from the confinement boundaries. In this case one can in principle add an infinite series of images (or solve Eq.2, with the correct boundary conditions, numerically or analytically using a Fourier representation) to obtain to a more isotropic-like solution. In our analysis such an addition it is not required because fast ions at energies such that $\delta \chi \sim 1$, are typically out of resonance with TAEs, a result that is confirmed by numerical analysis. For negative $\chi_{0}$ the model is essentially the same as what has been described, whereas for the case of perpendicular injection, i.e. $\chi_{0}<\chi_{s}$ the disctribution function can be constructed as $f_{\chi}=[\phi(\chi)+\phi(-\chi)] / 2$ for all $\chi$ regions.

The normalization function $C(r)$ in Eq.(1) is calculated by taking the radial profile of beam ion beta from the TRANSP analysis code and integrating $v^{2} f_{b} / 2$ over the velocity space to construct the local value of beta $\beta_{b}(r)$. We find $\beta_{b}(r)=\left(2^{3} \pi m_{b} / 3 B_{0}^{2}\right) \int v^{2} f d^{3} v$, which implies

$$
C(r)=\frac{3 B_{0}^{2} \beta_{b}(r)}{2^{5} \pi^{5 / 2} E_{b 0}}\left\{\frac{1}{2}+\frac{v_{*}^{2}}{6 v_{b 0}^{2}} \ln \left[\frac{\left(v_{b 0}+v_{*}\right)^{3}}{v_{b 0}^{3}+v_{*}^{3}}\right]+\frac{v_{*}^{2}}{\sqrt{3} v_{b 0}^{2}} \arctan \left[\frac{v_{*}-2 v_{b 0}}{\sqrt{3} v_{*}}\right]-\frac{\sqrt{3} \pi v_{*}^{2}}{18 v_{b 0}^{2}}\right\}^{-1}
$$

The above calculations were performed to lowest order aspect ratio where toroidicity effects are ignored. As we show this result is compatible locally with the one obtained by TRANSP in the equatorial plane, $\theta=0$. We expect that this result is also compatible with the TRANSP beam beta radial profiles, which is an average over the poloidal angle. This is because the NBI is close to tangential and the beam ion drift orbit radial width is small. 


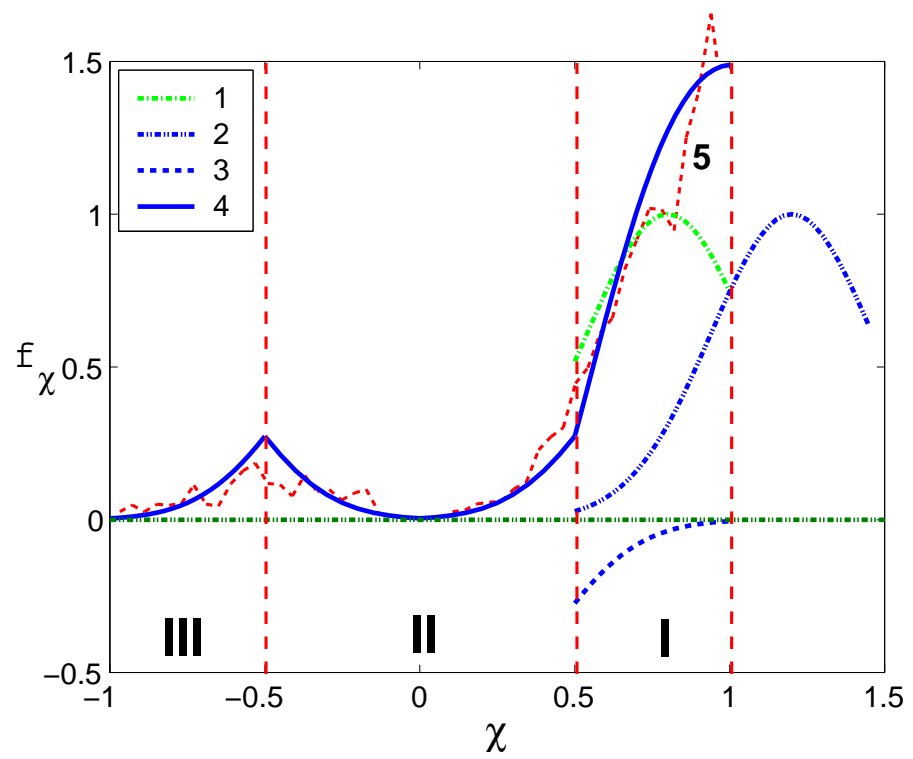

Figure 4: Results of modeling the beam ion pitch angle distribution function as given by Eq.(7) (curve 4) and its comparson with the TRANSP simulation (curve 5). The different pitch angle regions correspond to (I), co- passing beam ions; (II), trapped ions and (III), and counter- passing ions. In this case we used the low aspect ratio approximation for parameters $\delta \chi=0.37$ and $r / R=1 / 6$. Also shown are different terms for the model distribution function in region I: curves 1 and 2 correspond to first and second terms in the right hand side of Eq.(6), wheras curve 3 is the the sink term (second term of Eq.(7) for region I).

\section{TAE STABILITY ANALYSIS}

\section{A. NOVA eigenmode analysis}

In a previous numerical study Ref.[8] the domain of the most unstable TAE toroidal mode numbers $(n=7-20)$ in a nominal ITER plasma was computed using the global hybrid kinetic code NOVA-K. In this paper the fast ion beta estimate is somewhat lower than used in used Ref.[8] due to both a change in the injection geometry and the use of a better NBI confinement model in the TRANSP code. Hence, as the near-threshold stability analysis is sensitive to the fast ion beta we repeat the toroidal mode number scan similar to the one presented earlier. The results are shown in Fig. 5 for the nominal ITER plasma with on-axis NBI. We observe that due to the lower beam and alphas beta values the range of unstable $n$-values only spans from 7 to 12 for the case when both alpha particle and beam ion drives are included. It can be seen that TAEs are near threshold, and alpha particles alone do not seem be sufficient to destabilize TAEs. As a measure of 
how close we are to marginal stability we note that from table I (to be descussed hereafter) one can see that the growth rate from alphas and beams for $n=10$ is $\sim 1.5 \%$ of the eigenmode frequency compared to $\sim 0.3 \%$ for the predicted growth rate when damping is accounted for.

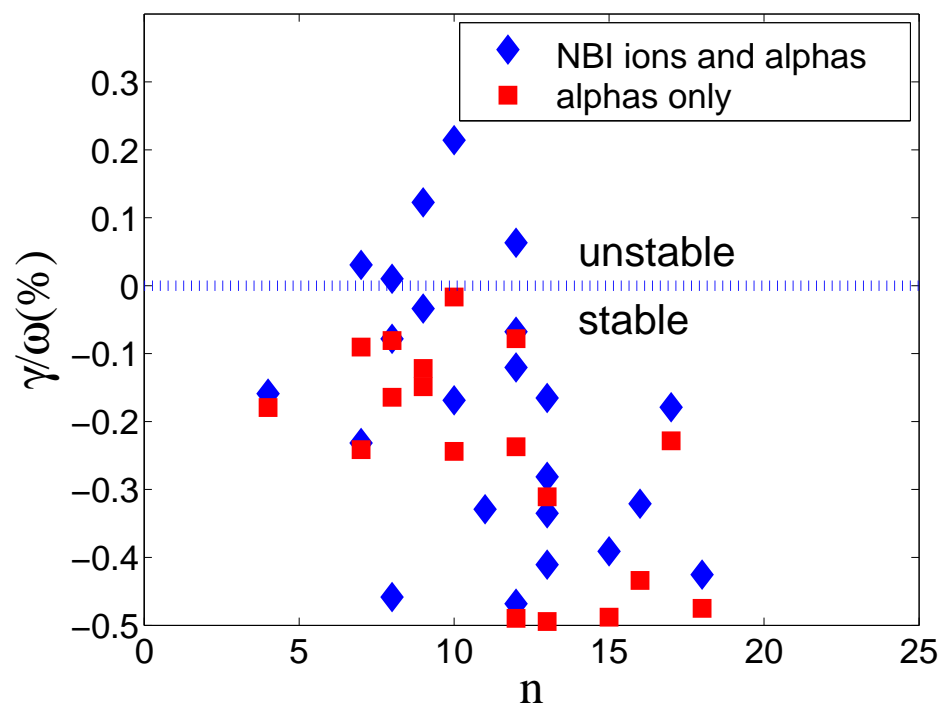

Figure 5: Toroidal mode number dependence of the TAE growth rates for the cases with the drive from alpha particles only (squares), and with the drive from both NBI ions and alpha particles (diamonds).

In this section we analyze in details the TAE stability for one of the most unstable toroidal numbers, $n=10$, in order to examine the sensitivity of the NBI drive to the injection characteristics, such as geometry and energy. The toroidicity and ellipticity induced gaps in the Alfvén continuum are calculated and are shown in figure 6 for the nominal ITER plasma discharge with $0.55 m$ off-axis NBI $[7,14]$. The normalized frequency is $\Omega=\omega q_{1} R_{0} / v_{A 0}$, where $v_{A 0}$ is the central Alfvén velocity, $q_{1}$ is the edge value of the safety factor, and $R_{0}$ is the major radius of the geometrical center. The center of the TAE gap envelope is proportional to the dependence $1 / q \sqrt{n_{e}}$, so that in tokamaks often the gap is nearly aligned along the minor radius, thereby allowing the TAE mode to span along a large portion of the minor radius without strongly interacting with the continuum. This was observed in present day experiments when a more open gap corresponds to MHD activity in the TAE frequency range (see for example Ref.[19-21]), whereas the plasma with the closed gap did not show such activity. The TAE gap in Fig. 6 is aligned except at the very edge due to strong variation of the safety factor towards the edge and flat density profile. This may result in TAE damping for global low- $n$ modes. In the present work the continuum damping is not included. 


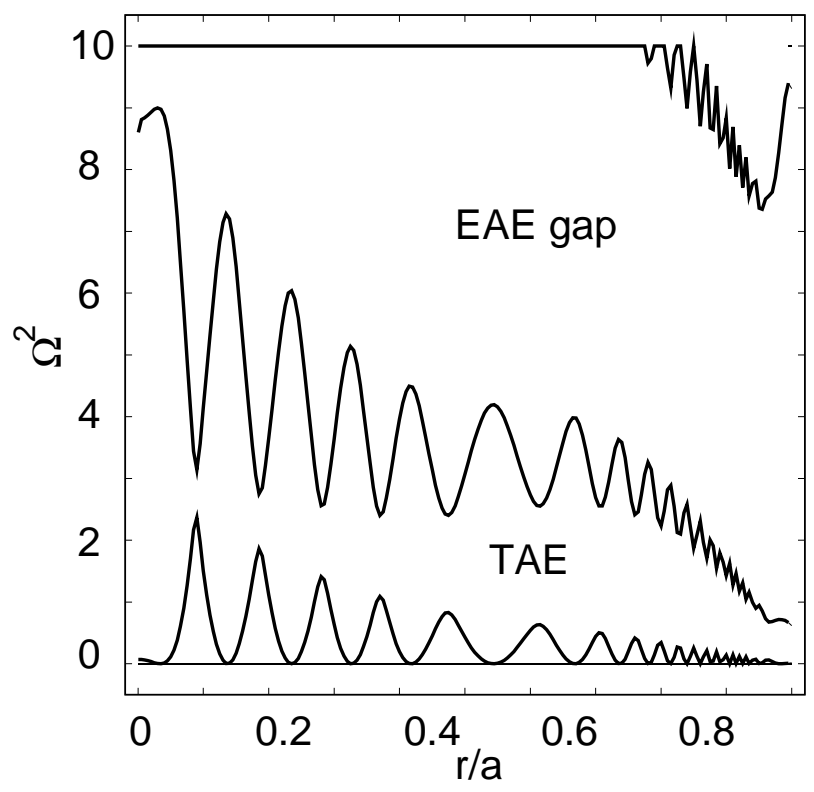

Figure 6: Alfvén continuum with gaps due to toroidal (TAE gap) and poloidal (EAE gap) coupling for $n=10$ as calculated by NOVA code.

The NOVA-K hybrid code predicts $n=10$ TAEs to be unstable in this ITER example if both the alpha particle, $\gamma_{\alpha}$, and beam ion, $\gamma_{\text {beam }}$, drives are combined. Among the various damping mechanisms that significantly reduce the drive are ion Landau damping, $\gamma_{i L a n d}$, radiative damping, $\gamma_{\text {rad }}$, and trapped electron collisional damping, $\gamma_{\text {ecoll }}$. The sum of damping and drive terms is plotted in figure 7 versus what we call the NBI impact parameter, $\Delta Z_{N B I}$, which is the vertical deviation from the magnetic axis of the beam injection line. For each case, up to two eigenmodes are unstable. The more the beam is directed off-axis the stronger the drive is. This is because during the on-axis NBI, beam ion beta builds up near the plasma center (see Fig.2), where the ion Landau damping is very strong. With the off-axis NBI the region of the strong beta gradient is shifted outward to the middle of the minor radius (for example $0.55 m$ off-axis, Fig. 2), so that both central ion Landau damping and edge trapped electron collisional damping are decreased. At the higher ion temperature case, $T_{i 0}=25.3 \mathrm{keV}$, the fusion beta is larger which enhances alpha particle destabilization of the TAE instability. However even in that case, where $\beta_{\alpha 0} \simeq 1.33 \%$, the pure fusion alpha-particle instability seems to still be marginally stable for $n=10$. However, with NBI present, the instability drive is strong enough to cause instability, with the growth rates for the most unstable mode at $\gamma_{\Sigma} / \omega=0.55 \%$. The contribution to the drive from alphas or beam ions is typically $\sim 1-3 \%$, which is with damping included, probably sufficiently small for the 
perturbation approach to be adequate.

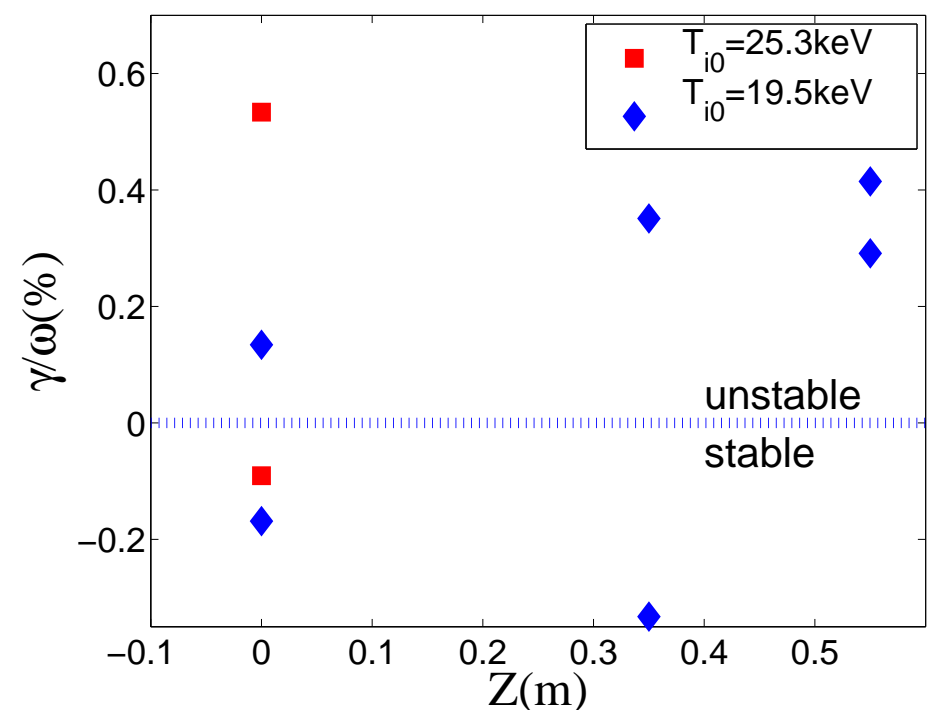

Figure 7: Found $n=10$ unstable TAE growth rates as functions of NBI impact parameter (vertical deviation from the magnetic axis).

As an example we present the stability properties of one of the most unstable $n=10$ TAEs for $0.55 \mathrm{~m}$ off-axis NBI. The radial structure of poloidal harmonics of this mode is presented in figure 8 versus the minor radius, which is defined as $r / a \equiv \sqrt{\psi / \psi_{0}}$, where $\psi$ is the poloidal magnetic flux, $\psi_{0}$ is the flux at the plasma edge, and we assumed $\psi=0$ at the magnetic axis. By comparing figures 6 and 8 one can notice that the mode is located in the gap with only a weak interaction with the continuum on the left. At that point its amplitude is small which means that continuum damping is expected to be small $[22,23]$. As the output of the TRANSP code shows, because of the neutral beam current drive, there is a low shear region at $0.4<r / a<0.6$ near the mode resonance with the Alfven continuum, where $s<0.3$. This implies that the "propagation" of TAE "couplets" into the center can be weak, with the result of low continuum damping $\gamma / \omega<0.001$ [23]. Table I lists the calculated damping mechanisms and drive terms due to alpha particles and beam ions at $\mathcal{E}_{b 0}=1 \mathrm{MeV}$. Though the beams are at lower beta value than the alpha particles, their drive is comparable due to their anisotropy at a fixed energetic particle energy density as is discussed in the next section. Note that three damping mechanisms are equally important for TAE stability, whereas as the calculations show at high $n$ 's $(n>15)$ the radiative damping becomes dominant and stabilizes the modes. 


\begin{tabular}{|c|c|c|c|c|c|c|}
\hline$\Omega^{2}$ & $\gamma_{\text {ecoll }} / \omega(\%)$ & $\gamma_{\text {iLand }} / \omega(\%)$ & $\gamma_{\text {rad }} / \omega(\%)$ & $\gamma_{\alpha} / \omega(\%)$ & $\gamma_{\text {beam }} / \omega(\%)$ & $\gamma_{\Sigma} / \omega(\%)$ \\
\hline 0.96 & -0.18 & -0.61 & -0.43 & 0.82 & 0.71 & 0.31 \\
\hline
\end{tabular}

Table I: Damping and driving growth rates of one of the most unstable $n=10$ TAE in the case of $0.55 m$ off-axis NBI. Given also is the TAE normalized eigenfrequency.

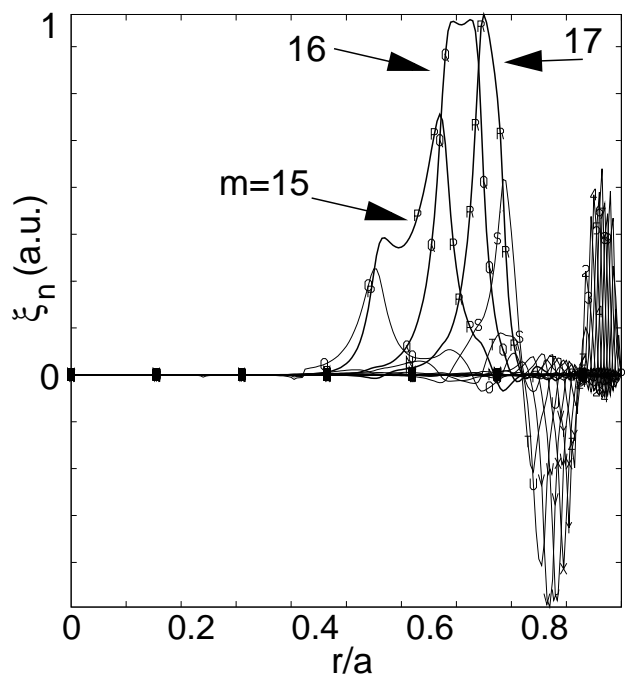

Figure 8: Radial mode structures of poloidal harmonic of the radial plasma displacement for one representative TAE mode with $\Omega^{2}=0.96$ in the case of $0.55 m$ off-axis NBI.

\section{THEORY OF NBI ANISOTROPY EFFECT ON TAES}

The anisotropy effect on the TAE drive has been studied for fast ions with beam-like anisotropic distributions [24] and for fusion products such as alphas with natural anisotropy due to finite orbit width effects $[25,26]$. For simplicity let us present the calculation of the drive in case of beam ion anisotropic distribution where there is a delta function pitch angle dependence of the form,

$$
f_{b} \simeq \frac{3 \beta_{\alpha} B^{2}}{2^{4} \pi^{2} E_{\alpha 0} v^{3}} \theta\left(v_{\alpha 0}-v\right) \delta\left(\chi-\chi_{0}\right)
$$

where $\theta$ is the step function. Note, that in the approximation of strongly passing beam ions (i.e. $\chi_{0} \sim 1$, which is reasonable for the tangential beam injection in ITER) $\chi$ is not changing along the particles's drift trajectory. For the simplicity we take a zero orbit radial width for the beam ion trajectories. Then the growth rate for the shear Alfvén instability we have [27]

$$
\frac{\gamma}{\omega} \simeq-\frac{v_{A}^{2} m_{b}^{2} \pi^{2}}{2 \omega R^{2} B^{2} E_{b 0}} \int d^{3} v\left[-\frac{E_{b o} \partial}{\partial E}-\frac{\omega_{*}}{\omega}\right] f_{b}\left(\frac{v_{\perp}^{2}}{2}+v_{\|}^{2}\right)^{2} \delta\left(\omega-\left(k_{\|} \pm \frac{1}{q R}\right) v_{\|}\right)
$$


where $\omega_{*}$ is the diamagnetic frequency of beam ions. This can be integrated directly and the expression for the growth rate reads

$$
\frac{\gamma}{\omega} \simeq \frac{-3 \pi \beta_{b} q^{2}}{8} \frac{v_{A}^{2}}{v_{b 0}^{2}} \frac{\left(1+\chi_{0}^{2}\right)^{2}}{\chi_{0}^{2}}\left[3+\frac{\chi_{0}^{2}}{\left(1+\chi_{0}^{2}\right)^{2}} \frac{\partial}{\partial \chi_{0}}\left(\frac{\left(1-\chi_{0}^{2}\right)\left(1+\chi_{0}^{2}\right)^{2}}{\chi_{0}^{3}}\right)-\frac{2 v_{A}^{2}}{v_{b 0}^{2}} \frac{\omega_{*}}{\chi_{0}^{2} \omega}\right]
$$

which can be transformed to

$$
\frac{\gamma}{\omega} \simeq \frac{-3 \pi \beta_{b} q^{2}}{8} \frac{v_{A}^{2}}{v_{b 0}^{2}} \frac{\left(1+\chi_{0}^{2}\right)^{2}}{\chi_{0}^{2}}\left[3-\frac{\left(3-2 \chi_{0}^{2}+3 \chi_{0}^{4}\right)}{\chi_{0}^{2}\left(1+\chi_{0}^{2}\right)}-\frac{2 v_{A}^{2}}{v_{b 0}^{2}} \frac{\omega_{*}}{\chi_{0}^{2} \omega}\right]
$$

Appropriate expressions can also obtained for the case where the beam orbit width is important. In

this case the above results are altered by multiplying Eq.(10) with a factor $\Delta_{b} / \Delta_{m}$, where $\Delta_{b}=q \rho_{\alpha}$ is the passing beam ion orbit width, and $\Delta_{m}=r_{m}^{2} / m s R$ is the TAE mode radial width scale [24]. In that case the expression for the growth rate is in a plateau regime with the respect to TAE mode number. It follows from Eq.(10) that the growth rate increases if the injection angle decreases, for example, if $\chi_{0}$ changes from $\chi_{0}=1$ to a lower value. This is confirmed by the numerical simulations presented in the next section. Note that the additional anisotropy term (second term in Eq.(10) is destabilizing and it considerebly reduces the stabilizing first term. For example at $\chi_{0}=1$ the sum of the first two terms in the square brackets of Eq.(10) is smaller then the first term by a factor $1 / 3$. For $\chi_{0}<1$ the anisotropy term increases faster than the first term in Eq.(10) so that at $\chi_{0}<0.8$ it becomes larger then the first one.

\section{NBI ANISOTROPY EFFECT CALCULATED FROM NOVA}

The properties of the TAE instability drive due to the anisotropic beam ion distribution function are studied with the pitch angle dependence in the form of Eq.(6) and fixed $\delta \chi^{2}=0.05$ for figure 9 and $\chi_{0}=0.8$ for figure 10. From Fig. 9 one can notice that the drive depends more strongly on the injection pitch angle than on the width of the distribution. Also calculations show that the drive is stronger for more localized modes. The instability is driven by passing particles rather than by trapped ones for almost all modes. Hence at the perpendicular injection (with $\chi_{0}=0$ ) TAEs are stable.

The effect of the anisotropy of beam ion distribution function is also computed by separating each of the driving or damping terms of the growth rate: the $\omega_{*}$ driving term (third term in square bracket of Eq.(10)), velocity gradient stabilizing term (first term in square bracket of Eq.(10)), and anisotropy driving term (second term in square bracket of Eq.(10)). We plot the growth rate as 


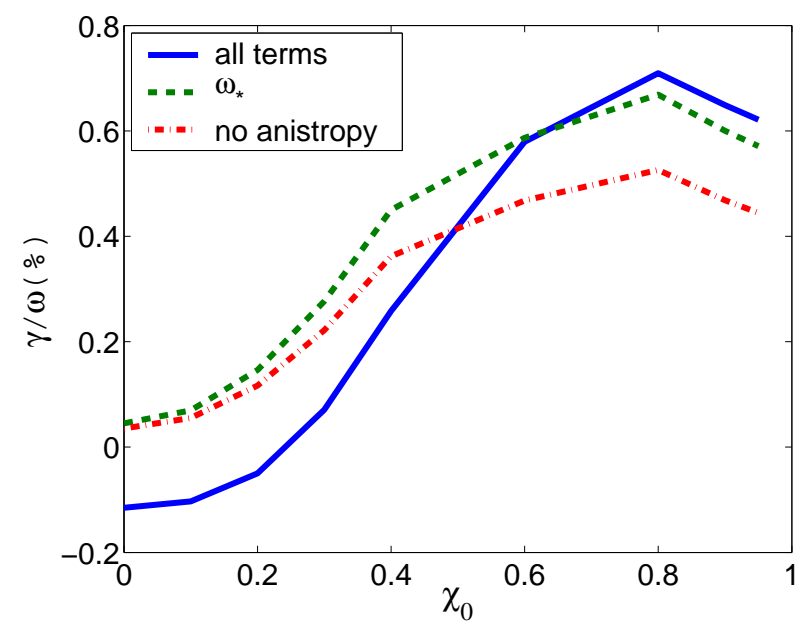

Figure 9: Pitch angle, $\chi_{0}$, (at fixed $\delta \chi^{2}=0.05$ ) dependence of different driving terms in the TAE growth rate. The solid curve is for all the driving terms, whereas the dashed curve is for velocity plus spatial gradient terms and the dot-dashed curve is for the spatial gradient term alone.

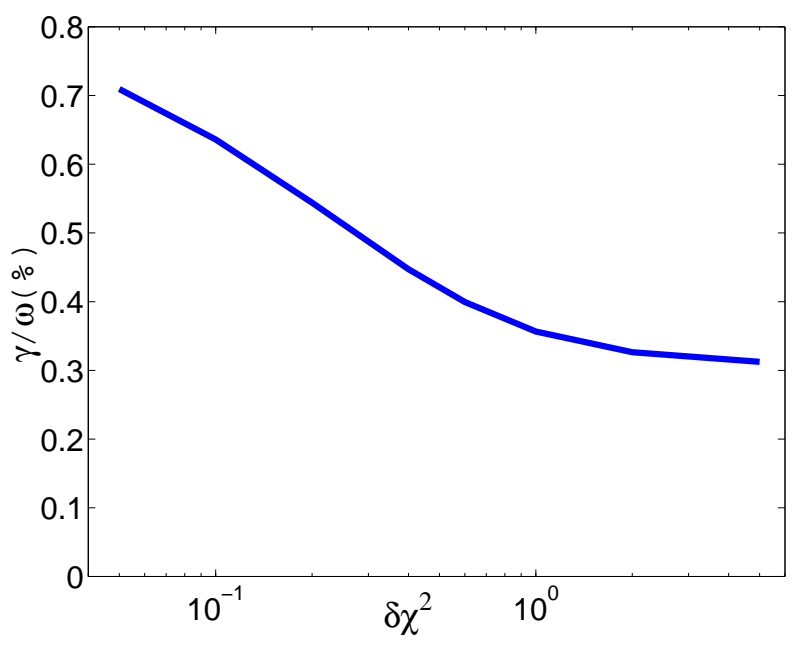

Figure 10: The growth rate dependence versus the beam ion distribution parameter $\delta \chi^{2}$ taken for $\chi_{0}=0.8$.

a function of $\chi_{0}$ including (i) all terms, (ii) spacial gradient drive ( $\omega_{*}$ curves), and (iii) velocity plus spacial gradient (no anisotropy curves) as indicated in figure 9. The anisotropy contribution accounts for $30-40 \%$ of the drive for the tangential injection $\chi_{0} \sim 1$. It becomes stabilizing for the perpendicular injection, $\chi_{0}=0$. Surprisingly from Fig. 9 it follows that the energy derivative alone, i.e. the anisotropy drive plus the velocity drive contribute to the destabilisation of the TAE. This seems to contradict the result of Eq.(10), where first and second terms in square bracket relate 
to each other as -3 to 2 , so that the net contribution is stabilizing. This is because the distribution function adopted in the derivation of the analytical expression does not include the critical velocity $v_{*}$. If it is included, which is the case for the numerical analysis, the velocity and the anisotropy drives are related as follows $-3 v^{3} /\left(v^{3}+v_{*}^{3}\right)$ to 2 . So that for ITER in which $v_{*} \sim v_{A}$ we find that ratio to be $\sim-1.5$ to 2 so that energy derivative alone is destabilizing in this case.

Since TAE excitation is due to the particle mode resonance it is expected that the instability will be sensitive to the injection energy. Such injection energy scan is shown in figure 11. Instability due to the beam ions is stabilized at beam energy $E_{b 0}<200 \mathrm{keV}$. Such low energy may not be enough to achieve the NBI goal of current drive and auxiliary heating because the beam may not penetrate deep enough [8]

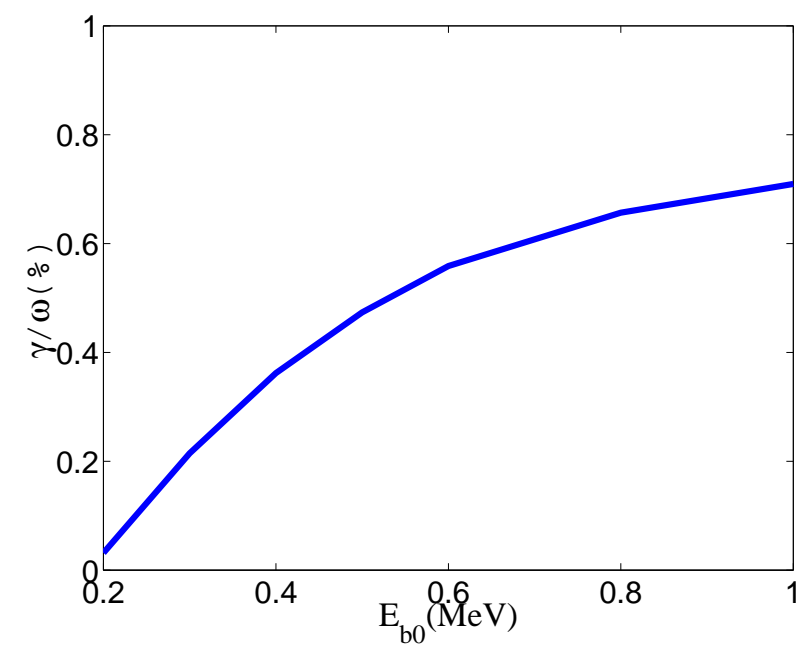

Figure 11: NBI energy dependence of the drive of two TAE modes.

\section{RELAXATION OF FAST ION PROFILES IN MULTIPLE TAE UNSTABLE PLASMA}

Finally we present a model for assessing the effect of TAE instabilities on the fast ions. In particular we want to develop a methodology for assessing in the case of instability, whether there is likely to be a substantial loss of energetic particles due to diffusion from the fields generated by the TAE modes.

In Ref. [8] the critical alpha particle pressure gradient was estimated from the balance between the energetic particle drive and the likely most important dissipative mechanisms, which were ion Landau damping and trapped electron collisions. Indeed, it was also shown that these two damping 
mechanisms are dominant for the expected radial location of the most unstable TAEs $r / a \gtrsim 0.5$. Equating the drive to damping terms gives,

$$
\frac{\partial \beta_{\alpha c r}}{\partial r}=-\frac{\gamma_{i L}+\gamma_{e c o l l}}{\gamma^{\prime} \alpha},
$$

where $\gamma_{\alpha}^{\prime}=\gamma_{\alpha} /\left(\partial \beta_{\alpha} / \partial r\right)$. Note that the right hand side of this equation depends on the background plasma parameters and is independent of $\beta_{\alpha}$.

We found that when there was on-axis NBI, we could accurately fit the $\alpha$-particle equilibrium steady state beta profile calculated by the TRANSP predictive run to a beta profile of the form, $\beta_{\alpha}=0.008\left[1-(r / a)^{2}\right]^{5}$. In the estimations in this section the plasma ion temperature profile is taken to be of the form $T_{i}(k e V)=20\left[1-(r / a)^{2}\right]$, and while the background beta is taken as, $\beta_{p c}=0.06\left[1-(r / a)^{2}\right]$. If the system is unstable (i.e. $\left.\left|\partial \beta_{\alpha} / \partial r\right|>\left|\partial \beta_{\alpha c r} / \partial r\right|\right)$, we assume that the TAE excitation will lead to radial diffusion of alpha particles with the transport described within the framework of a quasilinear theory.

The basic quasi-linear model is presented in the Appendix. We shall assume that the unstable region lies within a single radial band. Alpha particle transport is expected to flatten the alphas beta profile beyond the unstable region of linear theory and a new alpha particle beta profile $\beta_{\alpha r l x}(r)$ will form. To find the region to which that alpha particles spread to and $\beta_{\alpha r l x}(r)$, we note that the quasi-linear theoretical model predicts that the original unstable region spreads in space to satisfy the condition, $\partial \beta_{\alpha r l x} / \partial r=\partial \beta_{\alpha c r} / \partial r$ in a region $r_{1}<r<r_{3}$ and at these interfaces, $\beta_{\alpha r l x}\left(r_{1,3}\right)=$ $\beta_{\alpha}\left(r_{1,3}\right)$. Our task is to find $r_{1}$ and $r_{3}$ in accord with the procedure described in the Appendix. There it is also shown that there will be a point $r_{2}$ between $r_{1}$ and $r_{3}$ that satisfies $\beta_{\alpha r l x}\left(r_{2}\right)=$ $\beta_{\alpha}\left(r_{2}\right)$. These previous assertions assume that $r_{1}$ and $r_{3}$ will be found to lie within the boundaries of the plasma. Now, the original beta profile, $\beta_{\alpha}(r)$, is determined by classical processes which balances fusion energy production with the alpha particle drag to the plasma. In the relaxed state the beta profile remains $\beta_{\alpha}(r)$ outside the relaxed region, while the beta profile is at marginal stability within the relaxed region. Hence within the relaxed region, we find $\beta_{\alpha r l x}(r)=\beta_{\alpha}\left(r_{1}\right)+$ $\int_{r_{1}}^{r}\left(\partial \beta_{\alpha c r} / \partial r^{\prime}\right) d r^{\prime}$. As $\beta_{\alpha r l x}\left(r_{3}\right)=\beta_{\alpha}\left(r_{3}\right)$, one of the conditions to determine the endpoints is, $\beta_{\alpha}\left(r_{3}\right)-\beta_{\alpha}\left(r_{1}\right)=\int_{r_{1}}^{r_{3}}\left(\partial \beta_{\alpha c r} / \partial r^{\prime}\right) d r^{\prime}$. The additional condition is determined by the balance of classical processes with the quasi-linear diffusion of quasi-linear relaxation process in the TAE turbulent region. The Appendix indicates that applying a particle conservation condition in this region gives a reasonable estimate for this balance. Hence the end-points of the turbulent region is determined by the additional condition $\int_{r_{1}}^{r_{3}} \beta_{\alpha}\left(r^{\prime}\right) r^{\prime} d r^{\prime}=\int_{r_{1}}^{r_{3}} \beta_{\alpha r l x}\left(r^{\prime}\right) r^{\prime} d r^{\prime}$. We also note that 
the actual energy density that is redistributed is smaller than the above estimate. Only a fraction, $\eta$, of the alpha particle beta profile undergoes redistribution due to resonant interaction with TAE modes. In Refs.o $[29,30]$ this fraction was estimated ad $\eta=\left(v_{\alpha 0}-v_{\|}\right) v_{\|} / v_{\alpha 0}^{2} \leq 0.25$ for the straight cylinder geometry, where $v_{\|}$is particle parallel velocity resonant with TAE. As a result in the relaxation region the beta profile can be expressed as $\hat{\beta}_{\alpha}(r)=\eta \beta_{\alpha r l x}(r)+(1-\eta) \beta_{\alpha}(r)$. Thus the resulting alpha profile is given by

$$
\hat{\beta}_{\alpha}(r)=\left\{\begin{array}{c}
\beta_{\alpha}(r), r<r_{1} \\
\eta \beta_{\alpha r l x}(r)+(1-\eta) \beta_{\alpha}(r), r_{1}<r<r_{3} . \\
\beta_{\alpha}(r), r>r_{3}
\end{array}\right.
$$

In Fig. 12 we show the result of the relaxation of the beta profile with $\eta=0.25$. Also to demonstrate the sensitivity to the critical beta (and thus to the damping rate model) we show in addition an alpha particle profile obtained for the critical beta from Eq. (11) multiplied by 0.7, which is denoted $\hat{\beta}_{\alpha 1}$. For the latter case alphas are found to be redistributed up to the plasma edge with $4 \%$ losses. For figure 12 we note that the TAEs are locally unstable within $0.39<r / a<0.72$. After the quasi-linear transport model is applied alphas are redistributed within $r_{1} / a=0.30<$ $r / a<r_{3} / a=0.89$ (see curve marked with $\hat{\beta}_{\alpha}$ ) in Fig.12.

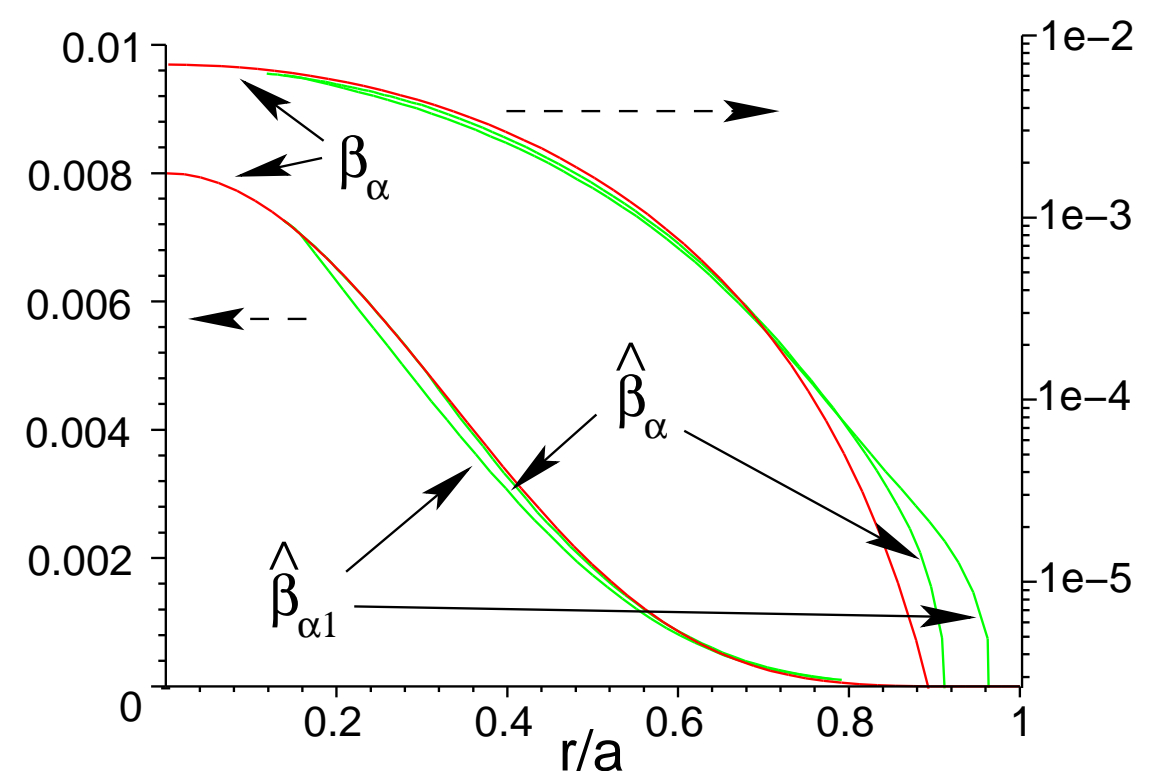

Figure 12: Alpha particle beta profiles initial and reconstructed using expressions for the local critical beta Eq.(11). Beta profile $\hat{\beta}_{\alpha 1}$ is obtained with the critical beta from Eq. (11) multiplied by 0.7. All the profiles are shown in linear and logarthmic scales. 
Stronger radial transport is expected if the thermal ion temperature is raised. This is because the fusion alpha-particle beta depends on $T_{i}: \beta_{\alpha} / \beta_{p c}=\sigma^{2} 0.117 T_{i}^{5 / 2} /(1+\sigma)$, where $\sigma \equiv\left(n_{D}+n_{T}\right) / n_{e}=0.8$. Figure 13 shows the expected loss dependence with increased alpha particle beta as the temperature was increased from a baseline case of $T_{i 0}=20 \mathrm{keV}, \beta_{p c 0}=6 \%$, and $\beta_{\alpha 0}=0.8 \%$. Here, in one case, we fixed the plasma density (solid curve) and in another case, the plasma beta (dashed curve), while keeping fixed $r_{3}=a$. In the case of fixed plasma density the predicted TAE induced transport is weaker because the ion Landau damping is increasing with ion temperature. With the constant beta, the ion temperature was varied from $20 \mathrm{keV}$ at $\beta_{\alpha 0}=0.8 \%$ to $\sim 24 \mathrm{keV}$ at $\beta_{\alpha 0}=1.3 \%$, whereas at fixed density the corresponding temperature range was from $20 \mathrm{keV}$ to $\sim 23 \mathrm{keV}$. We see that losses can become severe with increased temperature especially for the fixed beta case (note that MHD considerations may limit the operational beta to a fixed value).

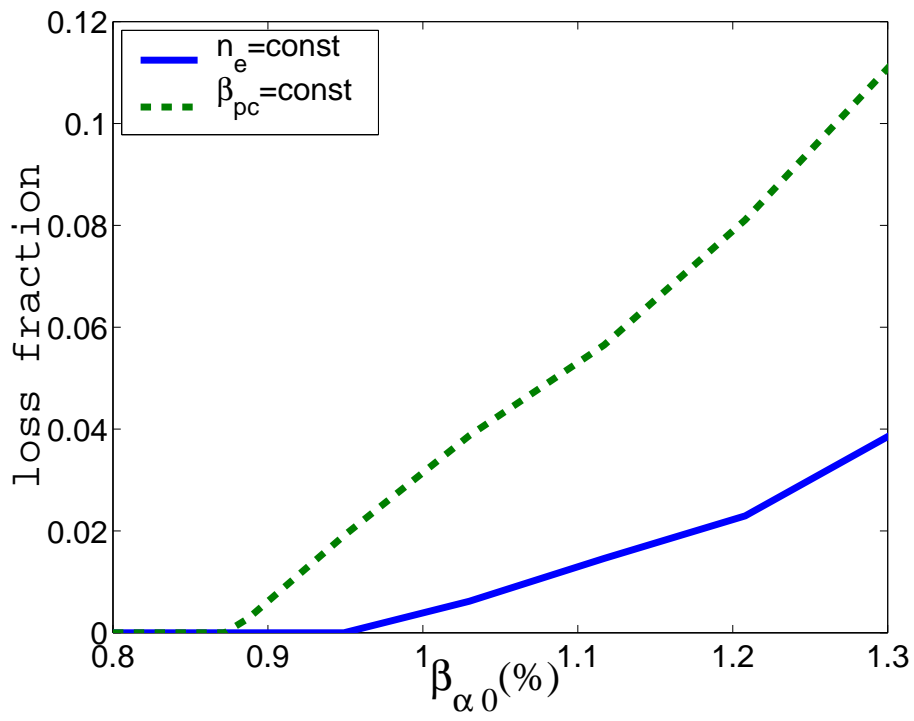

Figure 13: Expected alpha particle losses are shown as function of increased $\beta_{\alpha 0}$ keeping fixed total plasma beta (dashed curve, $\beta_{p c}+\beta_{\alpha}=$ const in which $\beta_{\alpha 0} \sim T_{i 0}^{5 / 2}$ ) and density (solid curve, $\beta_{\alpha 0} \sim T_{i 0}^{7 / 2}$ ).

In the calculations in this section we neglected the TAE interaction with beams assuming that only one specie is driving the instability. It should be possible to renormalize the critical gradients proportional to the contribution of each specie to the total drive. The model we propose needs to be corrected by numerical calculations to account for extra damping mechanisms. More detailed investigations can be adjusted to numerically evaluated damping and growth rates, as is obtained in NOVA-K. Our model shows that in the case of local instability theory the TAE instability ef- 
fects allows the window of operation in ITER but will establish the high temperature limit. The quasilinear theory applicability condition, which requires the overlap of particle resonances, must also be considered and this is a subject of further study.

\section{DISCUSSION}

We showed that NBI with tangential injection geometry is likely to destabilize TAEs in an ITER-like plasma. With perpendicular injection and/or lower beam energy one can reduce the TAE drive. Since the reactor plasma is supposed to be self-sustained without the beams, TAEs in a ITER-size machine will be driven only by alpha particles. As also follows from our previous study TAEs appear to be weakly unstable at operation where central temperatures are near $20 \mathrm{keV}$. On

the other hand NBI may provide an important tool for the experimental study of different types of AE instabilities by creating additional drive. Thus it is important to plan the NBI to be as flexible as possible in order to be able to change the conditions of AE excitation, i.e. by changing the geometry angle, energy, specie etc.

A quasi-linear model for alpha particle TAE induced transport has been developed. A critical assumption of the applicability of the quasilinear calculation is that resonance overlap of the TAE modes at the predicted level of the theory is enough to produce mode overlapping. This issue needs to be a subject of further study. If the overlap assumption is valid, then when we account for the growth rates from NOVA-K calculations the expected alpha particle profiles from Fig.(12) seems reasonable. It follows that even in the pessimistic case of local instability the effect of TAEs will be tolerable at temperatures comparable to $20 \mathrm{keV}$. However, the model predicts deleterious confinement conditions at somewhat higher central temperature $(\sim 23 \mathrm{keV})$. More verification is needed of this quasilinear model, but potentially it is an important tool for predictive codes such as TRANSP to be used to evaluate the effects of TAE driven transport on reactor performance. In principle one can determine the domain of plasma reactor parameters that can be achieved even with TAE unstable modes present.

\section{ACKNOWLEDGMENTS}

Discussions with A.Polevoi have been valuable for ITER plasma modeling. This work was supported by the United States Department of Energy under Contracts No. DE-AC02-76CH03073 
and DE-FG03-96ER-54346.

\section{Appendix A:}

We seek a steady state solution to a 1-d model we use to describe the energetic particle relaxation due to the TAE instability, where the density gradient causes the universal instability drive to be present. We use the model developed in Ref. [28]. This model considers the linear modes to be extremely localized, but continuously spread out in space. At each radial point the diffusion coefficient $D$, which is proportional to the mean square value of the field amplitude, satisfies the evolution equation,

$$
\frac{\partial D(x, t)}{\partial t}-2\left[\gamma_{L}(x, t)-\gamma_{d}(x)\right] D(x, t)=0
$$

where $\gamma_{d}$ is the damping rate of the mode due to background dissipation and $\gamma_{L}$ is the instability drive of the wave proportional to the radial spatial gradient of the distribution function. In this simplified model we take,

$$
\gamma_{L}(x, t)=-\sigma \frac{\partial f(x, t)}{\partial x}
$$

where $f$ is the time averaged distribution function for the energetic particles, $x$ a normalized radial variable and $\sigma$ is a positive constant. The distribution function satisfies the quasi-linear equation that results from the diffusion arising from the unstable spectrum and the relaxation of the energetic particles from classical processes. The classical relaxation is taken to satisfy a simple Krook collision operator with a relaxation rate $v$ and with a source present with of the form $v(x) f_{0}(x)$. The kinetic equation for the system is:

$$
\frac{\partial f}{\partial t}-\frac{\partial}{\partial x} D(x) \frac{\partial f}{\partial x}=v(x)\left(f_{0}-f\right)
$$

Clearly when $D=0$, the steady solution is $f=f_{0}$, and this solution can be sustained when there is stability everywhere in space so that $\gamma_{d}>\gamma_{L} \equiv-\sigma \partial f_{0} / \partial x$.

Suppose now $\gamma_{L}>\gamma_{d}$ in a spatial interval $x^{-}<x<x^{+}$. In that case the diffusion coefficient grows and attempts to flatten the distribution function in the unstable region. However, the flattening causes gradients to steepen at the interface between the original stable and unstable regions. 
As a result the unstable region broadens beyond the interval between $x^{-}$and $x^{+}$. The final distribution is expected to have the form of Fig.14 where the relaxation band spreads out to a larger interval. The region of relaxation stops only where the diffusion coefficient vanishes at the points $x_{u}$ and $x_{l}$. The distribution will be continuous at the points $x_{u}$ and $x_{l}$ so that the shape of the $f_{0}\left(x_{0}\right)$ is such that we will have an intermediate point $x_{0}$ where $f\left(x_{0}\right)=f_{0}\left(x_{0}\right)$, as can be seen in Fig.14.

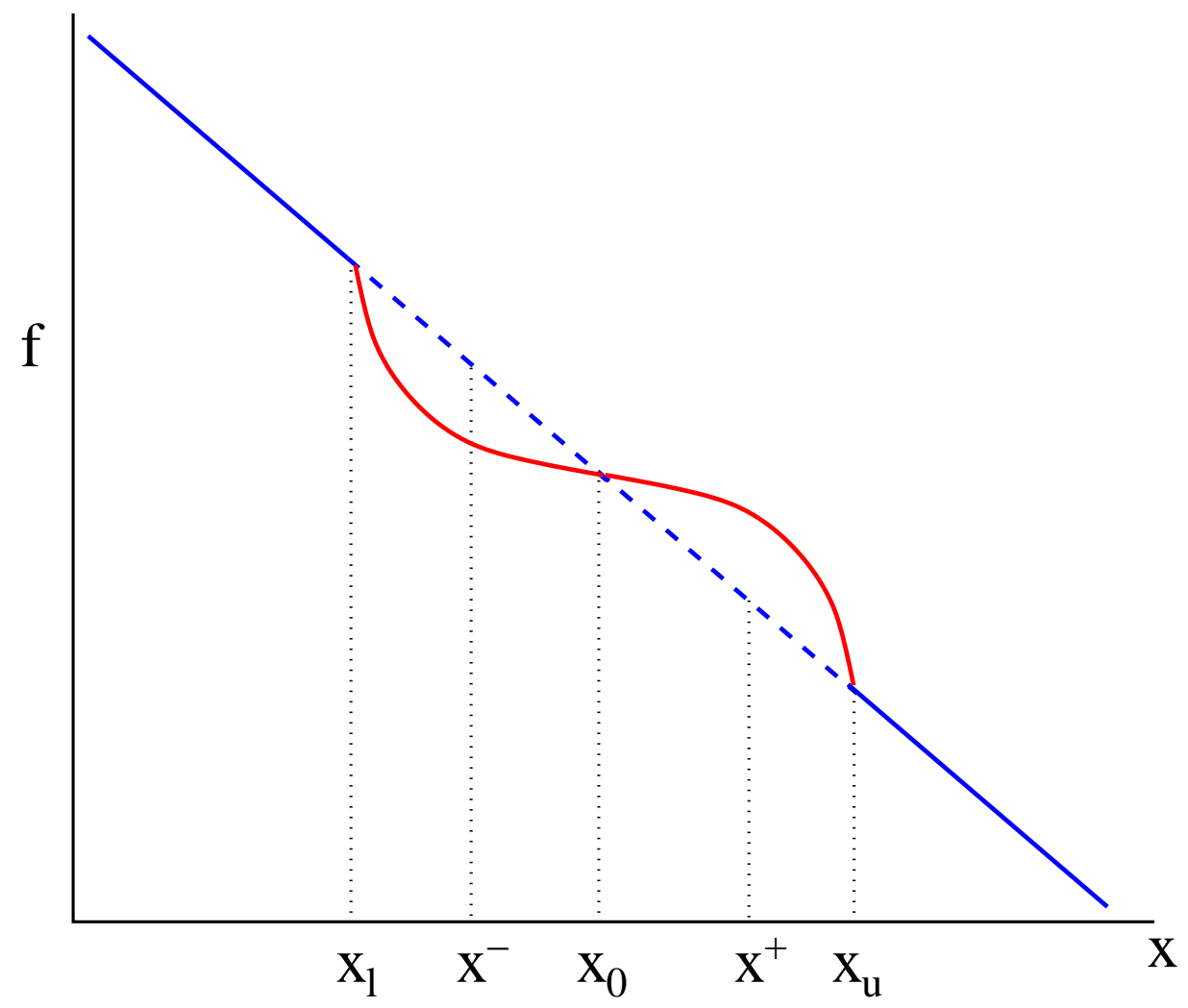

Figure 14: Relaxation of an initial equilibrium to the marginal stable one. Notation is explained in the text.

We now solve Eqs. (A1,A2,A3) in steady state to determine the final relaxed region. We can choose a suitable normalization that allows us to take $\sigma=1$. In this case we find as steady solutions,

$$
\frac{\partial f_{0}}{\partial x}=-\gamma_{L}, \frac{\partial f}{\partial x}=-\gamma_{d}
$$

and the relation,

$$
\frac{\partial D \gamma_{d}}{\partial x}=-v(x) \int_{x_{0}}^{x}\left[\gamma_{L}\left(x^{\prime}\right)-\gamma_{d}\left(x^{\prime}\right)\right] d x^{\prime}
$$

Now integrating Eq. (A4) with the condition that $D(x)$ vanishes at the end points $x=x_{l}, x_{u}$, yields the relations, 


$$
\begin{gathered}
D(x) \gamma_{d}(x)=D\left(x_{0}\right) \gamma_{d}\left(x_{0}\right)-\int_{x_{0}}^{x} v\left(x^{\prime}\right) \int_{x_{0}}^{x^{\prime}}\left[\gamma_{L}\left(x^{\prime \prime}\right)-\gamma_{d}\left(x^{\prime \prime}\right)\right] d x^{\prime \prime}, \\
D\left(x_{0}\right) \gamma_{d}\left(x_{0}\right)=\int_{x_{0}}^{x_{u}} d x^{\prime} v\left(x^{\prime}\right) \int_{x_{0}}^{x^{\prime}}\left[\gamma_{L}\left(x^{\prime \prime}\right)-\gamma_{d}\left(x^{\prime \prime}\right)\right] d x^{\prime \prime}, \\
\int_{x_{l}}^{x_{u}} d x v(x) \int_{x_{0}}^{x}\left[\gamma_{L}\left(x^{\prime}\right)-\gamma_{d}\left(x^{\prime}\right)\right] d x^{\prime}=0 .
\end{gathered}
$$

Note that Eq. (A5) implies that $D(x) \gamma_{d}(x)$ is maximum at $x_{0}$, as at that point $\gamma_{L}\left(x_{0}\right)>\gamma_{d}\left(x_{0}\right)$. The points $x_{l}, x_{0}$, and $x_{u}$ are determined from Eqs. (A8) and the two relations,

$$
\int_{x_{0}}^{x_{l, u}}\left[\gamma_{L}(x)-\gamma_{d}(x)\right] d x=0
$$

This last equation follows from the equality of $f$ and $f_{0}$ at $x=x_{l}, x_{0}$, and $x_{u}$ and the $\mathrm{x}$-integration of equations (A4).

As an example, consider the case where $f_{0}$ has a constant slope so that $\gamma_{L}$ is a constant and $\gamma_{d}$ has the form $\gamma_{d}=\gamma_{d 0}\left[1+a\left(x-x_{0}^{\prime}\right)^{2}\right]$, with $\gamma_{L}>\gamma_{d 0}$. The original unstable region is contained within the band $x^{-}<x<x^{+}$, where

$$
x^{ \pm}=x_{0}^{\prime} \pm\left(\frac{\gamma_{L}-\gamma_{d 0}}{a \gamma_{d 0}}\right)^{1 / 2}
$$

The solutions for the end points $x_{l}, x_{0}$, and $x_{u}$ are found from Eqs.(A8) and (A9) to be, $x_{u, l}-x_{0}^{\prime}=$ $\pm\left(6 \frac{\gamma_{L}-\gamma_{d 0}}{a \gamma_{d 0}}\right)^{1 / 2}, x_{0}=x_{0}^{\prime}$. Thus the unstable region is considerably broadened, but if this region is within the boundaries of the plasma, the diffusion does not lead to excessive energetic particle loss.

We observe that this theory gives a very simple way to estimate the energetic particle relaxation due to instability, and it is readily generalized to more complicated systems. In the text we take the radial coordinate as $x=r^{2} / 2$, and apply this result to the expected $\gamma_{L} \sim \gamma_{d}$ that arises in linearized TAE theory.

There are several important points that are in need further investigation that is pertinent in assessing the robustness of this procedure. One is to note that in the physical problem is in more than one phase space dimension. As result, even in the spatially unstable region, only a fraction of the particles that are slowing down in velocity space would have resonantly interacted with the wave and relaxed its distribution in the manner described in this theory. Kolesnichenko [29, 30] has estimated that in a burning plasma the fraction, $\eta$, of relaxed particles in the unstable region 
$\eta \sim 0.25$. Another important point is that quasi-linear model that has been used is based on the assumption that the basic TAE modes cause resonant particle-mode overlap, so that the particles can diffuse stochastically in the unstable region. However, we see from Eq. (A7), that the diffusion coefficient is proportional to the relatively weak classical relaxation rate $v$. As result it is possible that the mode amplitudes of the finite number of TAE modes that is predicted to be excited in a steady state solution are not large enough to achieve the mode overlap that is needed to justify the analysis. In this case it has been pointed out in Ref. [28] that rather than a steady behavior, bursty behavior arises where the instantaneous mode amplitude, and hence diffusion, is much larger than for the steady state prediction. This is an important subtle issue that will be addressed in later work. Yet another issue is the effect of finite orbit size in a tokamak plasma and how it is enhanced by field ripple for alpha particles near the edge. This effect is particularly important if the unstable region is predicted to move out to near the plasma edge.

[1] C. Z. Cheng, L. Chen, M. S. Chance, Ann Phys. (NY) 161, 21 (1985).

[2] C. Z. Cheng and M. S. Chance, Phys. Fluids 29, 3695 (1986).

[3] C. Z. Cheng, G. Y. Fu, and J. W. Van Dam, Princeton Plasma Physics Laboratory Report, PPPL-2585 (January, 1989), 14pp. in Proceedings of the Joint Varrena-Lausanne Workshop on Theory of Fusion Plasmas, Lausanne, Switzerland, Oct. 3-7, 1988, p. 259-270.

[4] G. Y. Fu and J. W. Van Dam, Phys. Fluids B 1, 1949 (1989).

[5] K. L. Wong, Plasma Phys. Cont. Fusion 39, 2471 (1997).

[6] W. W. Heidbrink, G. J. Sadler, Nucl. Fusion 34, 535 (1994).

[7] D.J.Campbell, Phys. Plasmas 8, 2041 (2001).

[8] N. N. Gorelenkov, H. L. Berk, R.B. Budny, et. al. Nuclear Fusion 43, 594 (2003).

[9] C. Z. Cheng, Phys. Reports, 211, 1 (1992).

[10] N. N. Gorelenkov, C. Z. Cheng, G. Y. Fu, Phys. Plasmas 7, 2802 (1999).

[11] G. Y. Fu, R. Nazikian, R. Budny, and Z. Chang, Phys. Plasmas 5, 4284 (1998).

[12] R. Nazikian, G. Y. Fu, S. H. Batha, et.al., Phys. Rev. Lett. 78, 2976 (1997).

[13] G.J. Kramer, S.E. Sharapov, R. Nazikian, et.al, Phys. Rev. Lett. 92, 015001-1 (2004).

[14] R. V. Budny, Nucl. Fusion 42, 1383 (2002).

[15] http://www.iter.org/ 
[16] S. V. Putvinskij, in Reviews of Plasma Physics, Vol. 18 (KADOMTSEV, B. B., Ed.), Consultants Bureau, New York (1986) 239.

[17] H. L. Berk, W. Horton, M. N. Rosenbluth, and P. H. Rutherford, Nucl. Fusion 15, 819 (1975).

[18] C. Angioni, A. Pochelone, N. N. Gorelenkov, et.al, Plasma Phys. Control. Fusion 44, 205 (2002).

[19] S. Bernabei, R. V. Budny, E. D. Fredrickson, et.al., Nucl. Fusion 41, 513 (2001).

[20] N. N. Gorelenkov, S. Bernabei, C.Z. Cheng, et.al., Nuclear Fusion 40, 1311 (2000).

[21] G.J. Kramer, C.Z. Cheng, G.Y. Fu, Y. Kusama, R. Nazikian, T. Ozeki, and K. Tobita, Physical Review Letters 83, 2961 (1999).

[22] BERK, H. L., VAN DAM, J. W., GUO, Z., LINDBERG, D. M., Phys. Fluids B 4, 1806 (1992).

[23] ROSENBlUTH, M.N., BERK, H.L., VAN DAM, J.W., Z., LINDBERG, D.M., Phys. Fluids B 4, (1992) 2189.

[24] H.L. Berk, B.N. Breizman and H. Ye, Phys. Letters A 162, 475 (1992).

[25] N.N. Gorelenkov, Sov. J. Plasma Phys. 18, 289 (1992).

[26] N.N. Gorelenkov, Plasma Phys. Rep. 19, 404 (1993).

[27] Y.M. Li, S.M. Mahajan, D.W. Ross, Phys. Fluids 30, 1466 (1987).

[28] H.L. Berk, Breizman, J. Fitzpatrick, H.V. Wong, Nucl. Fusion Nucl. Fusion 35, 1661 (1995).

[29] Ya.I. Kolesnichenko, Nucl. Fusion, 20, 727 (1980)

[30] V.S. Belikov, Ya.I. Kolesnichenko, V.V. Lutsenko, Nucl. Fusion, 35, 207 (1995). 



\section{External Distribution}

Plasma Research Laboratory, Australian National University, Australia

Professor I.R. Jones, Flinders University, Australia

Professor João Canalle, Instituto de Fisica DEQ/IF - UERJ, Brazil

Mr. Gerson O. Ludwig, Instituto Nacional de Pesquisas, Brazil

Dr. P.H. Sakanaka, Instituto Fisica, Brazil

The Librarian, Culham Laboratory, England

Mrs. S.A. Hutchinson, JET Library, England

Professor M.N. Bussac, Ecole Polytechnique, France

Librarian, Max-Planck-Institut für Plasmaphysik, Germany

Jolan Moldvai, Reports Library, Hungarian Academy of Sciences, Central Research Institute for Physics, Hungary

Dr. P. Kaw, Institute for Plasma Research, India

Ms. P.J. Pathak, Librarian, Institute for Plasma Research, India

Ms. Clelia De Palo, Associazione EURATOM-ENEA, Italy

Dr. G. Grosso, Instituto di Fisica del Plasma, Italy

Librarian, Naka Fusion Research Establishment, JAERI, Japan

Library, Laboratory for Complex Energy Processes, Institute for Advanced Study, Kyoto University, Japan

Research Information Center, National Institute for Fusion Science, Japan

Dr. O. Mitarai, Kyushu Tokai University, Japan

Dr. Jiangang Li, Institute of Plasma Physics, Chinese Academy of Sciences, People's Republic of China

Professor Yuping Huo, School of Physical Science and Technology, People's Republic of China

Library, Academia Sinica, Institute of Plasma Physics, People's Republic of China

Librarian, Institute of Physics, Chinese Academy of Sciences, People's Republic of China

Dr. S. Mirnov, TRINITI, Troitsk, Russian Federation, Russia

Dr. V.S. Strelkov, Kurchatov Institute, Russian Federation, Russia

Professor Peter Lukac, Katedra Fyziky Plazmy MFF UK, Mlynska dolina F-2, Komenskeho Univerzita, SK-842 15 Bratislava, Slovakia

Dr. G.S. Lee, Korea Basic Science Institute, South Korea

Institute for Plasma Research, University of Maryland, USA

Librarian, Fusion Energy Division, Oak Ridge National Laboratory, USA

Librarian, Institute of Fusion Studies, University of Texas, USA

Librarian, Magnetic Fusion Program, Lawrence Livermore National Laboratory, USA

Library, General Atomics, USA

Plasma Physics Group, Fusion Energy Research Program, University of California at San Diego, USA

Plasma Physics Library, Columbia University, USA

Alkesh Punjabi, Center for Fusion Research and Training, Hampton University, USA

Dr. W.M. Stacey, Fusion Research Center, Georgia Institute of Technology, USA

Dr. John Willis, U.S. Department of Energy, Office of Fusion Energy Sciences, USA

Mr. Paul H. Wright, Indianapolis, Indiana, USA 
The Princeton Plasma Physics Laboratory is operated by Princeton University under contract with the U.S. Department of Energy.

\author{
Information Services \\ Princeton Plasma Physics Laboratory \\ P.O. Box 451 \\ Princeton, NJ 08543
}

Phone: 609-243-2750

Fax: 609-243-2751

e-mail: pppl_info@pppl.gov

Internet Address: http://www.pppl.gov 